\title{
Estrategias tecnológicas en bibliotecas universitarias como centros de recursos para la investigación y el aprendizaje en la región Caribe de Colombia
}

\author{
Hugo G. Hernández-Palma1, William Niebles-Nuñez ${ }^{2}$, Carlos Pacheco-Ruiz ${ }^{2}$ y Claudia Rojas-Martínez ${ }^{2}$ \\ (1) Universidad del Atlántico, Programa Administración de Empresas, Barranquilla-Colombia. \\ (correo-e: hugohernandezpalma@gmail.com) \\ (2) Universidad de Sucre, Programa de Administración de Empresas, Sincelejo-Colombia. \\ (correo-e: william.niebles@unisucre.edu.co; carlos.pacheco@unisucre.edu.co; claudia.rojas@unisucre.edu.co)
}

Recibido Feb. 18, 2020; Aceptado Abr. 17, 2020; Versión final Jun. 17, 2020, Publicado Dic. 2020

\begin{abstract}
Resumen
La presente investigación estuvo dirigida a caracterizar las estrategias tecnológicas utilizadas en bibliotecas universitarias como centros de recursos para la investigación y el aprendizaje en la región Caribe Colombiana. La metodología de la investigación fue de tipo descriptiva, con diseño no experimental de tipo transversal. La población estuvo conformada por 23 empleados de las bibliotecas universitarias. La técnica de recolección de datos se llevó a cabo a través de un cuestionario estructurado con alternativas tipo escala Likert. Para el cálculo de la confiabilidad se utilizó el método de alfa Cronbach, donde se obtuvo un valor de 0.79 . Los resultados muestran que el $52 \%$ de los encuestados estuvo totalmente de acuerdo con los medios de almacenamiento de información, un $36 \%$ de acuerdo, un $9 \%$ consideró estar indiferente, y solo un $4 \%$ en desacuerdo. Se concluye que existe la necesidad de realizar actividades orientadas a desarrollar habilidades y destrezas.
\end{abstract}

Palabras clave: tecnologías de información; biblioteca; recursos de investigación; educación

\section{Technological strategies at university libraries as a resource center for research and learning in the Colombian Caribbean region}

\begin{abstract}
This research study aimed to identify the technological strategies used at university libraries as resource centers for teaching and learning in the Colombian Caribbean region. The research methodology was descriptive, with a non-experimental cross-sectional design. The population consisted of 23 university library employees. The data collection technique developed was through a questionnaire, structured with Likert scale alternatives. The alpha Cronbach method was used for reliability calculation and a value of 0.79 was obtained. The results showed that $52 \%$ of the surveyed subjects strongly agreed with the type of equipment used for data storage, $36 \%$ agreed, $9 \%$ were indifferent, and only $4 \%$ disagreed. It is concluded that there is a need for activities aimed at developing skill sets.
\end{abstract}

Keywords: information technology; library; research resources; education 


\section{INTRODUCCIÓN}

En la actualidad las tecnologías de información y comunicación (TIC) se han originado en una serie de escenarios, surgidos a partir del proceso de globalización; orientando a los individuos a la búsqueda de mejores formas de ejecutar los procesos: uno de ellos es cómo gestionar el conocimiento que forma parte del interés de los usuarios en el momento requerido. Dentro de este marco, el manejo de las TIC, en cualquier ámbito incluye contar con una habilidad integral, en función de usar todos los modos de trasmitir, comprender y recibir ideas, pensamientos, como parte de conocimiento, puede ser verbal, escrita, electrónica, para transferir e intercambiar dicha información. Por esto, el desarrollo tecnológico, la globalización y el uso habitual de las computadoras, ha dado paso a la digitalización de la información, facilitando el acceso a la información.

Manly, Leonard y Riemenschneider (2014) sostienen que la sociedad actual se encuentra integrada a las leyes y actuaciones impuestas por las tecnologías de la información y de la comunicación (TIC). Las mismas están vinculadas al procesamiento de diferentes mensajes a partir de una señal digital de carácter numérico, transmitido por diversas tecnologías, en la que subyace fundamentalmente el ordenador como la máquina capaz de procesar este tipo de información. Dentro de estas perspectivas, hay organizaciones que requieren de ciertas plataformas para la atención al usuario, las mismas depende del manejo y control tecnológico de quienes ofrecen el servicio al usuario, en este sentido atender las peticiones de los estudiantes, para Martínez et al (2017), permite el desarrollo del pensamiento crítico, a partir del material disponible en las bibliotecas, pero también de lo existente a escala mundial es una imperiosa necesidad hoy en día.

En este orden de ideas, Bosco y Rodríguez (2011), exponen que los nuevos procesos tecnológicos y la era de la información han modificado los escenarios educativos, las actitudes, además del uso y alcance de dicha información y, por ende, la forma en la cual se ha de gestionar, en virtud de dar soporte a los procesos del contexto digital. En este sentido el área de bibliotecas se encuentra inmiscuida en un período de conmoción y expectación. Por ende, las herramientas para la localización de información en Internet, así como las fuentes de información disponibles en la red, permiten que los usuarios cuenten con una infinidad de nuevos servicios, con un gran valor agregado, como los hiperdocumentos referenciales, que contienen una lista de recursos de información en Internet, localizados, evaluados, descritos y compilados por un bibliotecario-referencista, para un individuo o grupo de usuarios con un perfil común.

Aunado a ello, se percibe la realidad inmediata de las bibliotecas la cual ha creado nuevas formas de organizar, almacenar, recuperar, transmitir y adquirir información concebida como base fundamental para la gestión del conocimiento, la misma ha influido en las maneras de enseñar, aprender, investigar, generando nuevos servicios de información. Además de ello, las bibliotecas como unidades de información han contemplado en su haber información impresa donde el sistema de gestión se sustenta en la información estática, monolítica, se analizan diferentes aspectos intrínsecos, para ser utilizada por las personas, en entornos físicos, este sistema diseñado como objetos que tenían límites claros, en virtud del deterioro generado en el tiempo.

A este respecto, Mačiulytè y Gaile (2014) consideran que las TIC, son herramientas y procesos para acceder, recuperar, guardar, organizar manipular, producir, intercambiar además presentar información por medios electrónicos, incluyen hardware, software, telecomunicaciones en la forma de computadores y programas tales como aplicaciones multimedia, así como sistemas de bases de datos. En este sentido, expresan Martínez, Steffens, Ojeda, y Hernández (2018), que la tecnología se sustenta en el paso de lo analógico a lo digital, ello facilita la comprensión de grandes cantidades de información en memorias cada vez más pequeñas, con gran capacidad de almacenamiento, ello ha permitido enriquecer los catálogos con nuevas prestaciones y funcionalidades, asimismo al incorporarse a la web, se busca satisfacer la demanda de información de los usuarios del internet.

Además de lo expuesto, existe otra fase de este proceso, orientada a la creación de servidores institucionales los cuales tienen la función de asumir la responsabilidad de la gestión de esa información digital generada en las instituciones de educación superior del mundo, donde se debe gestionar los textos completos, actualizados y relevantes, en virtud de poner a disposición del usuario todo el material necesario, como es expuesto por Hernández et. al., (2018). En este sentido, Cabero, Vázquez y López (2018), sostienen que la realidad educativa y tecnológica en las aulas de los diferentes niveles académicos, en nuestro sistema educativo en la actualidad, viene de la mano de la incorporación de nuevas herramientas que acerquen a los alumnos y alumnas, de forma sencilla, lúdica y formativa, a los contenidos curriculares. Una de las tecnologías que toman mayor impulso e importancia en la actualidad es la realidad aumentada, la cual se ha ido abriendo camino.

No obstante, a pesar de no cubrir con las expectativas y requerimientos de los usuarios, las TIC han servido para agilizar los procesos bibliotecarios, aunque no han mejorado la gestión universitaria en términos de gestión de información y gerencia del conocimiento, por ende, en algunas áreas existe carencia de un pensamiento estratégico en gestión de información y conocimiento. Se hace necesario buscar nuevos modelos que hagan factible erradicar estas debilidades, por lo tanto, se viene trabajando en diferentes 
instituciones de educación superior de la Región Caribe de Colombia. En este sentido, las herramientas de tecnológicas enlazadas con los medios de almacenamiento, las redes y las bases de datos electrónicas se relacionan con las nuevas propuestas tecnológicas promoviendo el fácil acceso desde cualquier ordenador.

Dentro de este marco, el impulso en las demandas de información originadas por una sociedad en constantes transformaciones, para Hernández et al (2018), se promueve en las unidades de información adscritas a las instituciones de educación superior, específicamente de la región Caribe Colombiana, una propuesta innovadora que facilite el acceso a la red de información nacional e internacional, desarrollando así las nuevas tendencias en lo que a gestión de servicios se refiere; se soportan mediante el uso y demanda de las tecnologías de información y comunicación TIC, expuesto por Martínez, Steffens, Ojeda y Hernández (2018). De acuerdo con lo planteado los investigadores se formularon la siguiente interrogante ¿cómo se caracterizan las estrategias tecnológicas utilizadas en bibliotecas universitarias como centros de recursos para la investigación y el aprendizaje en la región Caribe Colombiana?

Ante este desafío, existe la disyuntiva sobre cómo deben orientarse las estrategias tecnológicas en las instituciones de educación superior, destacando que se debe contar con medios de almacenamiento de última tecnología, desarrollo de redes académicas, de información y de relaciones de conocimiento, así como contar con arduas bases de datos electrónicas, para fomentar la integración de los contenidos de manera que sean capaces de ser verdaderos centros de recursos para la investigación y el aprendizaje en un entorno digital. Bajo este contexto, las repercusiones de estas transformaciones, así como las decisiones a este respecto deberán ser capaces de adaptarse a aplicaciones necesarias para el contexto universitario. Se puede, sin embargo, apuntar una serie de elementos para guiar dicha estrategia, cada uno de los cuales supone un reto en sí mismo para las bibliotecas universitarias. En tal sentido, la operatividad de los nuevos avances en TIC aplicados en el área de los servicios informativos de las bibliotecas universitarias se constituye en uno de los principales recursos que coadyuvan al mejoramiento continuo de los procesos, generando en el usuario la posibilidad de acceso rápido y oportuno a la información requerida, con el uso de estrategias tecnológicas en bibliotecas universitarias como centros de recursos para la investigación y el aprendizaje.

\section{OTROS ANTECEDENTES}

Para el desarrollo de este trabajo es conveniente caracterizar las estrategias tecnológicas con las que cuentan las plataformas digitales de las bibliotecas universitarias, tales como: medios de almacenamiento, redes y bases de datos electrónicas, exponiendo si tienen la capacidad para ser un recurso para la investigación.

\section{Plataforma tecnológica un desafío para la educación superior}

Respecto a la plataforma tecnológica, Krull y Duart (2017) manifiestan que en las últimas décadas han sido testigo de grandes cambios en el mundo bibliotecario. Particularmente se ha experimentado una modificación dramática en los servicios por el hecho de que los bibliotecarios ahora cuentan con una serie increíble de recursos electrónicos a su disposición para llevar a cabo su tarea. Asimismo, debe buscarse que la biblioteca del futuro sea una institución híbrida, las mismas deben contener colecciones digitales e impresas, asumiendo que será el bibliotecario navegador quien guiará a los usuarios de las bibliotecas hacia las fuentes más relevantes, abriendo camino al conocimiento, a la información contenida en las supercarreteras de la información. Este desarrollo ha sido especialmente ventajoso para las bibliotecas de países en desarrollo, en especial aquellas que no cuentan con grandes presupuestos para la adquisición de información y repertorios de referencia. En la actualidad el bibliotecario debe trabajar muy duro para organizar efectivamente esos recursos, promover el acceso fácil a los datos en línea, y al mismo tiempo enseñar a los usuarios cómo utilizar eficazmente la información, particularmente la contenida en las bases electrónicas durante el desarrollo de sus investigaciones. La aplicación de la tecnología de la información en las unidades de información se ha hecho bajo la premisa de realizar los mismos procesos, sin cambio, pero mediados por una máquina y en consecuencia no ha habido cambios significativos en sus actividades.

En la actualidad con relación a las colecciones, éstas pueden ser gerenciadas por una firma especializada fuera del campus universitario o hacerlo a través de una plataforma tecnológica con bases de datos, servicios de referencia y servicios de préstamo. En un futuro cercano, uno de los escenarios percibidos es la desaparición de los dos últimos servicios, dado el surgimiento y mejoramiento constante de instrumentos como la televisión interactiva, los teléfonos móviles de última generación, las búsquedas en lenguaje natural y controlado, Internet, Internet 2, correos electrónicos, grupos de discusión. En el mismo ámbito, Ng'ambi y Bozalek (2013) consideran que un aspecto a considerar es conocer la demanda de los centros de investigación de las instituciones de educación superior, para adquirir las bases de datos de interés para áreas específicas, esto además estén representadas en las colecciones impresas.

Asimismo, crear bases de datos que contemplen los estudios de usuarios y sus requerimientos de información, los títulos de suscripciones, editoriales comerciales, sociedades científicas, editoriales universitarias y gubernamentales impresas y en línea. Esto con la finalidad de organizar inteligentemente la 
información para que sea empleada efectivamente en la producción de nuevo conocimiento y en la formulación de políticas públicas. El conocimiento de la plataforma tecnológica en la institución es indispensable para el desarrollo y uso eficiente de los productos digitales. Por lo cual se hace relevante detallar algunos aspectos de las estrategias, entre ellas los medios de almacenamientos, las redes y las bases de datos electrónicas.

\section{Medios de almacenamiento}

La necesidad de publicar por parte de los creadores y de las bibliotecas para preservar y difundir el conocimiento se ve incrementada por el desarrollo de publicaciones digitales que aun cuando son menos con respecto a las publicaciones en papel durante varios años las bibliotecas universitarias coexistirán con colecciones híbridas, pero el profesional de la información tiene un compromiso con el usuario real o virtual. Asimismo, con la creación de las unidades que manejan las plataformas tecnológicas, la universidad se convierte en una vía para la brecha informacional que tiene un país latinoamericano con relación a los países industrializados. El uso de la tecnología de la información supone otro problema, cómo llenar de contenidos esas redes, esto es parte de las políticas públicas de los países, aunado al ámbito institucional la respuesta parece estar en el diseño de sistemas que permitan capturar, organizar y difundir los grandes volúmenes de información. Dentro de este marco en las bibliotecas universitarias, se requiere contar con medios de almacenamiento actualizado, pero además de gran capacidad, los cuales deben ser proporcionales al tipo de redes existentes, de esta manera, capturar y difundir en forma coordinada la información.

\section{Trabajo en red}

Con respecto a la red, Novo, Varela y Ramil (2013), asumen que la ciencia en red es una potenciadora del trabajo en colaboración, el mismo está transformando el flujo de los procesos implicados en la comunicación científica. En un entorno de trabajo en red, para Viloria et al (2019), se debe ser capaz de soportar procesos en el cual varias personas, desde distintos ordenadores, trabajan en un espacio virtual de colaboración sobre un objeto, ello sustentado en el proceso de análisis e investigación pueden incorporar anotaciones, referencias a un artículo sobre ese objeto guardado en algún repositorio, acceder a una base de datos de un museo donde hay otras representaciones del objeto y referenciarlas, añadirle nuevos metadatos y una transcripción.

Para Hernández et al (2019), el sistema de redes posibilitaría integrar diferentes componentes que existen alrededor de la red, para así guardar el nuevo objeto resultante representando su naturaleza compleja y expresando las relaciones que mantienen esas partes entre sí, añadiendo semántica a esas relaciones de manera que puedan ser "entendidas" por las máquinas, luego podrán volver a procesar ese objeto en nuevas situaciones de investigación. Este objeto ha perdido su carácter estático y monolítico, convirtiéndose él mismo en una red de relaciones cuyos bordes son difusos, en un "documento" que representa una senda de relaciones que pueden ser reconstruidas de distintas formas por las máquinas. En este contexto, la investigación en red se desarrolla en entornos de colaboración distribuidos, por tal razón Tarute y Gatautis (2014), expresan que se requiere la existencia de una infraestructura de datos que presente esa información, como una representación de un objeto virtual global del conocimiento, disponible para ser procesado por las herramientas y aplicaciones de la "e-ciencia" de las comunidades virtuales en sus diferentes ámbitos científicos. Este corpus de información será una representación computarizada en sí misma que vive, crece e interactúa con sus creadores más que un registro o archivo pasivo de información.

Resulta importante conocer el papel que puede jugar la biblioteca y el bibliotecario: desde un punto de vista humanístico, se podría decir que la biblioteca sería el centro de la comunidad que garantizase el acceso democrático a esa información a aquellos que no tuvieran medios materiales para hacerlo. Hay que recordar que el fenómeno internet, a pesar de la notoriedad dada en prensa y medios de comunicación, afecta a una ínfima parte de la población. Ante este hecho para Sangrá (2010) la labor del bibliotecario es difícil de determinar mucho más, cuando los fenómenos tecnológicos se suceden vertiginosamente, la renovación es constante. Pero se podría aventurar que tendría una gran tarea en lo relativo a formación de usuarios, búsquedas bibliográficas especializadas y proporcionar información elaborada. Tampoco conviene adentrarse en el futuro, internet es una realidad que está ahí, en las bibliotecas empiezan actualmente a disponer de conexiones, con ello, ha de plantearse en qué medida se puede mejorar los servicios haciendo uso de esta nueva herramienta tecnológica. En este sentido, uno de los componentes del proceso de comunicación científica que también ha acusado los cambios en los procesos de investigación en la "e-ciencia" son los propios resultados de esa investigación, o lo que denominan Berrio, Pitre y Hernández (2018), "las publicaciones científicas", es decir, los resultados de una investigación que se publican en las revistas científicas.

En un estudio presentado por Dirks y Hey, (2007), se evidencia que el objeto que representa los resultados de la investigación de la "e-ciencia" incorpora además los datos que han servido de base para la investigación, las herramientas de software que se han utilizado para su análisis, interpretación, transformación, visualización y simulación, las especificaciones del hardware para transformar esos datos en conocimiento, 
la visualización en 3D, gráficos, diagramas, animaciones, junto con los datos textuales, notas, anotaciones, referencias bibliográficas (con el enlace al texto completo) y el artículo. Se requiere una herramienta que capture de forma precisa de dónde proviene cada una de las partes y encapsule sus diferentes componentes en un único "documento" compuesto que puede entonces ser guardado y publicado en un repositorio digital.

\section{Bases de datos electrónicas}

La biblioteca universitaria debe ser un centro de información, de forma tal que su misión se cumplirá si realiza gestión de información. La gestión de información, según Bustos y Colb, (2010), incluye selección, adquisición, arriendo, organización, control, diseminación y uso de información en todas sus variedades de fuentes y soportes, relevante para la efectiva operación de las organizaciones. Es decir, la gestión de información contempla desarrollar equilibrios entre recursos de información actual, además permanente, entre información local y virtual, entre recursos de información propios, así como el acceso a recursos contra demanda en el momento que se requieren, en la diversificación de soportes de información analógicos y digitales, mono y multimediales.

Dentro del concepto de gestión de recursos de información, Cai (2017), considera diferentes recursos, por ello la biblioteca universitaria no sólo como un agente que consume información elaborada por otros, sino también le asigna un rol protagónico en la sistematización y comunicación de los conocimientos necesarios para mejorar la disponibilidad de recursos para el estudio, así como el aprendizaje, la preservación y comunicación del conocimiento generado en la universidad, la mantención de la memoria intelectual de la institución y la preserva. En este sentido, la información actual bajo la perspectiva de Sánchez, García, Steffens, y Hernández (2019) es la información que representa el conocimiento comunicado, cuyo plazo de difusión es inferior al tiempo requerido para entregar al público un documento monográfico y cuya vigencia es más corta que la de aquél. Representa el estado del arte en todas las áreas del conocimiento. Abarca publicaciones periódicas primarias y secundarias, literatura gris, bases de datos, servicios de alerta, servicios de suministro de información contra demanda e información contenida en sitios web.

Así mismo, la información permanente es para Bustos y Colb (2010), aquella información bibliográfica, que por la forma en que se encuentra organizada, el tiempo que el autor tarda en producir la obra y la amplitud de los contenidos que cubre, tiene una vigencia superior a un año. Generalmente este tipo de documento no refleja el estado del arte, especialmente en las ciencias y la tecnología. Su condición de permanente es independiente del soporte físico o electrónico en que se encuentre almacenada. Este rol proactivo conduce a la biblioteca universitaria, entre otras tareas, a tener que liderar proyectos de digitalización de recursos bibliográficos dentro de un marco de respeto del derecho de autor y a participar en la fijación de políticas de comunicación del conocimiento generado en la universidad (edición de revistas científicas, tesis, entre otros). La gestión de recursos de información para Viloria et al (2019), consideran que el concepto de desarrollo de colecciones, facilidades de acceso a otras colecciones, adquisiciones cooperativas a través de consorcios, almacenamiento cooperativo, acceso electrónico a bases de datos y a otros recursos de información disponibles a través de las redes de telecomunicaciones. Dentro de estas perspectivas el mismo autor genera la importancia de buscar un equilibrio entre recursos de informaciones locales y virtuales. El equilibrio está dado en cada caso por las características de la institución, el adecuado soporte a los programas académicos y de investigación, por la oferta de información virtual a la cual tiene acceso. Para que los servicios virtuales existan según Bustos y Colb. (2010) se deben cumplir las siguientes condiciones expresadas en la Figura 1.
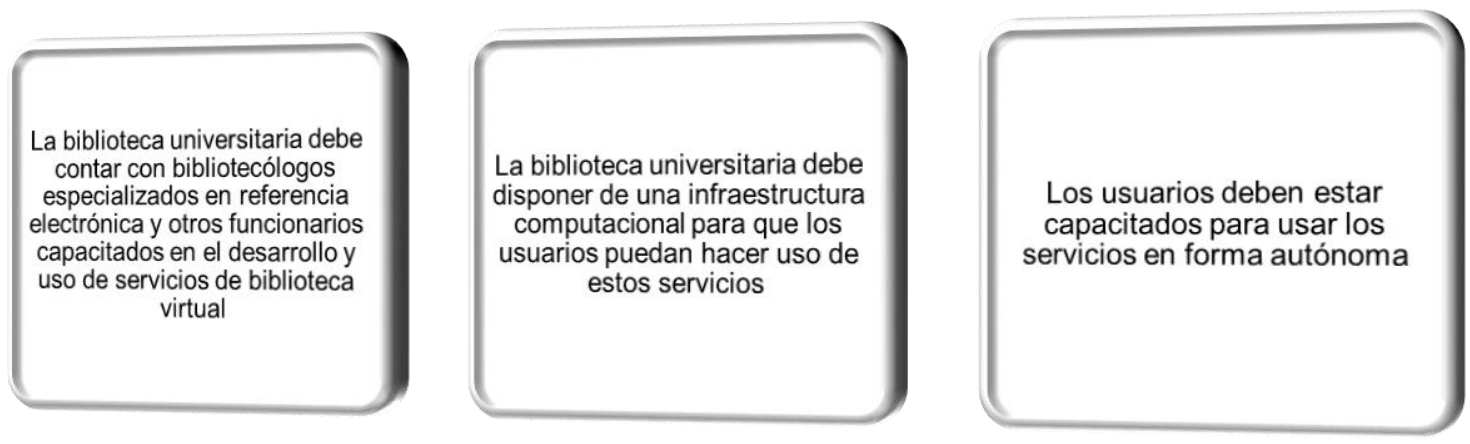

Fig. 1. Condiciones de los servicios virtuales universitarios. Adaptado de Bustos y Colb (2010)

Cabe destacar que sólo en la medida que las condiciones indicadas precedentemente se cumplan, es válido sustituir servicios presenciales por servicios virtuales, en este respecto la opinión de Villarreal, García, Hernández y Steffens (2019) es que la biblioteca universitaria debe tener autonomía suficiente para gestionar las adquisiciones bibliográficas, realizar un adecuado control presupuestario y, en general, realizar todas las operaciones necesarias para coordinar y supervisar la ejecución del programa de gestión de recursos de información. 


\section{METODOLOGÍA}

La investigación que se desarrolló está orientada bajo el método descriptivo, debido a que se pretendió caracterizar las estrategias tecnológicas utilizadas en bibliotecas universitarias como centros de recursos para la investigación y el aprendizaje en la región Caribe Colombiana, describiendo los elementos de la plataforma tecnológica como base de las estrategias. Aunado a ello, el estudio se percibe como una investigación de campo, dado que se recolectaron los datos en las bibliotecas seleccionadas para tal fin, para obtener información sobre la problemática existente. Se utilizó un diseño no experimental, en este ámbito las variables no fueron manipuladas por los investigadores, dado que la información que lo referencia se tomó tal y como se desarrollan en la realidad de los hechos. La recolección de datos se realizó en un único momento, obteniendo de esta forma una sola medición, por lo cual se determina como transversal. La población estuvo conformada por los coordinadores y empleados de las bibliotecas de la universidad del Atlántico y Universidad de Sucre Colombia, responsables de las actividades digitales, tales como manejo de redes, repositorio digital, control y manejo de los medios de almacenamiento y administración de la plataforma tecnológica, integrada (23) personas; esta distribución puede apreciarse en la Tabla 1.

Tabla 1. Distribución de población, Bibliotecas universitarias (2019)

\begin{tabular}{|l|l|}
\hline Instituto de educación superior & Unidad de análisis Empleados \\
\hline Universidad del atlántico & 12 \\
\hline Universidad de sucre & 11 \\
\hline Total Población & 23 \\
\hline
\end{tabular}

En esta investigación se utilizó un cuestionario de recolección de datos y el mismo fue respondido en forma directa por el personal que integra la población del estudio, veintitrés (23) empleados de las bibliotecas, convirtiéndolo de esta forma en un cuestionario de carácter simple. Se tuvo un censo poblacional debido a que se tomó en cuenta a todos los empleados de las bibliotecas de ambas universidades que trabajan exclusivamente las áreas digitales de estas, siendo 12 de la Universidad del Atlántico y 11 de la universidad de Sucre. El cuestionario estuvo comprendido con cuatro opciones de respuestas para que cada uno de los sujetos encuestados seleccionara la opción pertinente. La validación de los instrumentos de esta investigación se efectuó a través del juicio de expertos, una vez validado el cuestionario y realizadas las correcciones, se procedió a aplicar la prueba piloto, para proceder a determinar la confiabilidad de este. El paso siguiente fue calcular la confiabilidad, la cual se efectuó a través del coeficiente de Alfa Cronbach, este se aplica para los cuestionarios con escala tipo Likert, el resultado fue 0,79 . El instrumento utilizado se exhibe a continuación en la Tabla 2.

Tabla 2. Instrumento utilizado en el estudio

\begin{tabular}{|c|c|c|}
\hline Indicadores & \# & Cuestionamiento \\
\hline \multirow[t]{3}{*}{$\begin{array}{l}\text { Medios de } \\
\text { almacenamiento }\end{array}$} & 1 & $\begin{array}{l}\text { ¿Considera que los medios de almacenamiento de información, con los que cuenta en la } \\
\text { biblioteca cumple los requerimientos para el servicio al usuario? }\end{array}$ \\
\hline & 2 & ¿En la biblioteca están orientados a la actualización continua de las publicaciones digitales? \\
\hline & 3 & $\begin{array}{l}\text { ¿El sistema utilizado en la unidad de información permite organizar grandes volúmenes de } \\
\text { información? }\end{array}$ \\
\hline \multirow[t]{3}{*}{ Redes } & 4 & $\begin{array}{l}\text { ¿En la biblioteca donde Ud. labora, el trabajo en equipo es base de la investigación en } \\
\text { internet? }\end{array}$ \\
\hline & 5 & $\begin{array}{l}\text { ¿La internet le ofrece ventajas con respecto a la rapidez para obtener información, al } \\
\text { momento de ejecutar sus labores? }\end{array}$ \\
\hline & 6 & $\begin{array}{l}\text { ¿Se considera la existencia de una infraestructura de datos para respaldar la información en } \\
\text { la biblioteca? }\end{array}$ \\
\hline \multirow[t]{3}{*}{$\begin{array}{l}\text { Bases de datos } \\
\text { electrónicas }\end{array}$} & 7 & $\begin{array}{l}\text { ¿La biblioteca universitaria como centro de información digital cumple con los } \\
\text { requerimientos mínimos, para atender al usuario? }\end{array}$ \\
\hline & 8 & $\begin{array}{l}\text { ¿En la biblioteca se genera información en todas sus variedades de fuentes, para la } \\
\text { operación de las actividades de investigación? }\end{array}$ \\
\hline & 9 & $\begin{array}{l}\text { ¿Existe sistematización de los procesos para mejorar la disponibilidad de recursos para el } \\
\text { estudio y aprendizaje? }\end{array}$ \\
\hline
\end{tabular}

Por otra parte, el análisis de datos permite organizar toda la información en forma coherente y sencilla que facilité la generación de resultados, por ende, el análisis de los datos se efectúa sobré la matriz de datos utilizando un programa de computadora, en este mismo sentido, se utiliza la descripción de cada variable de forma separada y sus análisis estadísticos independientemente, con la finalidad de poder determinar la correlación existente entre ellas. Por ende, requieren de un tratamiento estadístico, el mismo depende del tipo de método de investigación seleccionado, de las operaciones que pueden ejecutar y de la escala de la variable. 


\section{RESULTADOS}

En la Tabla 3 que se aprecia a continuación, puede observarse la información referida al indicador "medios de almacenamiento", asociando los tres primeros ítems, donde el número 1 está referido a los medios de almacenamiento de información con los que cuenta la unidad sustentando si estos cumplen o no, los requerimientos necesarios; al cuál $52 \%$ de los encuestados estuvo totalmente de acuerdo, un $36 \%$ de acuerdo, además $9 \%$ consideró estar indiferente, solo $4 \%$ en desacuerdo. Con respecto al ítem número 2 , orientado a actualización continua de las publicaciones digitales, al respecto $43 \%$ de la población estuvo totalmente de acuerdo, un $36 \%$ de acuerdo, $17 \%$ se mostró indiferente; pero además $4 \%$ estuvo en desacuerdo. Por otro lado, el ítem 3 pretendió determinar si el sistema utilizado en la unidad de información permite organizar grandes volúmenes de información, a lo cual contestaron $56 \%$ de acuerdo, mientras $26 \%$ totalmente de acuerdo y finalmente, los restantes encuestados se repartieron en las opciones indiferente y en desacuerdo con $9 \%$ para cada una.

Tabla 3. Medios de almacenamiento

\begin{tabular}{|c|c|c|c|c|c|c|}
\hline \multirow{2}{*}{ Categorías İtems } & \multicolumn{2}{|l|}{1} & \multicolumn{2}{|l|}{2} & \multicolumn{2}{|l|}{3} \\
\hline & $\mathrm{Fa}$ & $\%$ & $\mathrm{Fa}$ & $\%$ & $\mathrm{Fa}$ & $\%$ \\
\hline Totalmente de acuerdo & 12 & 52 & 10 & 43 & 6 & 26 \\
\hline De acuerdo & 8 & 36 & 8 & 36 & 13 & 56 \\
\hline Indiferente & 2 & 9 & 4 & 17 & 2 & 9 \\
\hline En desacuerdo & 1 & 4 & 1 & 4 & 2 & 9 \\
\hline Muy en desacuerdo & 0 & 0 & 0 & 0 & 0 & 0 \\
\hline Total & 23 & 100 & 23 & 100 & 23 & 100 \\
\hline
\end{tabular}

Atendiendo a lo expuesto en la tabla 3 se concibe que en las bibliotecas se pueda contar con una plataforma tecnológica adecuada al contexto, sobre esto Sangrá (2010) manifiesta que las últimas décadas han sido testigo de grandes cambios en el mundo bibliotecario. Particularmente se ha experimentado una modificación dramática en los servicios electrónicos por el hecho de que los trabajadores bibliotecarios hoy en día cuentan con una amplia serie de recursos electrónicos a su disposición para llevar a cabo su tarea de promover y facilitar el acceso a la información.

En este sentido, los medios de almacenamiento, con los que cuenta las unidades en estudio cumplen los requerimientos, destacando que el uso de la tecnología de la información es parte de las políticas de las universidades en estudio, aunado al ámbito institucional ellas deben orientarse al diseño de sistemas que permitan capturar, organizar y difundir los grandes volúmenes de información. Por ende, en las bibliotecas universitarias, se requiere contar con medios de almacenamiento actualizado, pero además de gran capacidad, los cuales deben ser proporcionales al tipo de redes existentes, de esta manera, capturar y difundir en forma coordinada la información.

Siguiendo el análisis de la información, el indicador Redes (contemplado en la Tabla 4), incluye tres ítems. El primero de los ítems, el ítem número 4 está orientado hacia poder determinar si el trabajo en equipo es base de la investigación en internet, a lo cual los encuestados contestaron de acuerdo y en desacuerdo, 30\% para cada uno, mientras que las categorías totalmente de acuerdo e indiferente, se ubicaron $17 \%$, no obstante, solo $4 \%$ consideró estar totalmente en desacuerdo. El Ítem número 5, sugiere que el internet le ofrece ventajas con respecto a la rapidez para obtener información, al momento de ejecutar sus labores, al respecto, $39 \%$ estuvo de acuerdo, así como 34\% totalmente de acuerdo. En este orden de ideas, en las categorías indiferente y en desacuerdo se dividió la población restante eligiendo dichas opciones en proporción equitativa, es decir $13 \%$ para cada opción. Cabe destacar que no hubo elección alguna para la opción muy en desacuerdo. Por otra parte, la información expresada sobre el ítem número 6 muestran los resultados referidos a la existencia de una infraestructura de datos para respaldar la información en la biblioteca, a este respecto, 30\% de los encuestados estuvo de acuerdo, mientras que $26 \%$ se mostró indiferente, y 17\% estuvo en desacuerdo, asimismo que para las alternativas totalmente de acuerdo y muy en desacuerdo, la tendencia fue de $13 \%$ para ambas.

Tabla 4. Redes

\begin{tabular}{|c|c|c|c|c|c|c|}
\hline \multirow{2}{*}{ Categorías İtems } & \multicolumn{2}{|l|}{4} & \multicolumn{2}{|l|}{5} & \multicolumn{2}{|l|}{6} \\
\hline & $\mathrm{Fa}$ & $\%$ & $\mathrm{Fa}$ & $\%$ & $\mathrm{Fa}$ & $\%$ \\
\hline Totalmente de acuerdo & 4 & 17 & 8 & 34 & 3 & 13 \\
\hline De acuerdo & 7 & 30 & 9 & 39 & 7 & 30 \\
\hline Indiferente & 4 & 17 & 3 & 13 & 6 & 26 \\
\hline En desacuerdo & 7 & 30 & 3 & 13 & 4 & 17 \\
\hline Muy en desacuerdo & 1 & 4 & 0 & 0 & 3 & 13 \\
\hline Total & 23 & 100 & 23 & 100 & 23 & 100 \\
\hline
\end{tabular}


En cuanto a las redes, se afirma que la red de internet le ofrece ventajas con respecto a la rapidez para obtener información, al momento de ejecutar sus labores, además de contar con la existencia de una infraestructura de datos para respaldar la información en la biblioteca, a este respecto, Berrio, Pitre, y Hernández (2018), consideran que el sistema de redes posibilitaría la agregación de estos diferentes componentes sobre la red, así guardar el nuevo objeto resultante representando su naturaleza compleja expresando las relaciones que mantienen esas partes entre sí, añadiendo semántica a esas relaciones de manera que puedan ser entendidas por las máquinas, luego podrán volver a procesar ese objeto en nuevas situaciones de investigación. Para Bosco y Rodríguez (2011), el internet es la red de datos más extensa del mundo, por otra parte, para Krull y Duart (2017) la labor del bibliotecario es difícil de determinar, mucho más cuando los fenómenos tecnológicos se suceden vertiginosamente y la renovación es constante. Pero se podría aventurar que tendría una gran tarea en lo relativo a formación de usuarios, búsquedas bibliográficas especializadas y proporcionar información elaborada.

La Tabla 5, muestra el ultimo indicador bases de datos electrónicas, el cual involucra en primer término al ítem 7 que cuestiona si la biblioteca universitaria es un centro de información digital que cumple con los requerimientos mínimos, para atender al usuario. En este sentido la población contestó de la siguiente manera: $30 \%$ de la población estuvo de acuerdo, $26 \%$ se mostró indiferente, 17 , \% en desacuerdo, mientras que para las alternativas totalmente de acuerdo y totalmente en desacuerdo, la tendencia fue de $13 \%$ para ambas opciones. Por otra parte, en cuanto al ítem 8 acerca de generar información en todas sus variedades de fuentes, para la operación de las actividades laborales, un $26 \%$ estuvo en desacuerdo, $22 \%$ totalmente en desacuerdo, pero para alternativas totalmente de acuerdo, de acuerdo e indiferente, obtuvieron $17 \%$ para cada una de ellas. Finalmente, el ítem 9 el cual cuestiona respecto a la existencia de la sistematización de los conocimientos para mejorar la disponibilidad de recursos para el estudio y aprendizaje, $35 \%$ estuvo en desacuerdo, un $22 \%$ de acuerdo, además $17 \%$ se mostró indiferente, mientras que para las alternativas totalmente de acuerdo y totalmente en desacuerdo $13 \%$ para cada una.

Tabla 5. Bases de datos electrónicas

\begin{tabular}{|l|l|l|l|l|l|l|}
\hline \multirow{2}{*}{ Categorías } & \multicolumn{2}{|l|}{ Ítems } & \multicolumn{2}{l}{8} & \multicolumn{3}{l|}{9} \\
\cline { 2 - 8 } & Fa & $\%$ & Fa & $\%$ & Fa & $\%$ \\
\hline Totalmente de acuerdo & 3 & 13 & 4 & 17 & 3 & 13 \\
\hline De acuerdo & 7 & 30 & 4 & 17 & 5 & 22 \\
\hline Indiferente & 6 & 26 & 4 & 17 & 4 & 17 \\
\hline En desacuerdo & 4 & 17 & 6 & 26 & 8 & 35 \\
\hline Muy en desacuerdo & 3 & 13 & 5 & 22 & 3 & 13 \\
\hline Total & 23 & 100 & 23 & 100 & 23 & 100 \\
\hline
\end{tabular}

Sobre las bases de datos electrónicas, se constató que la biblioteca universitaria es un centro de información digital el cual cumple con los requerimientos mínimos, para atender al usuario, generan información en todas sus variedades de fuentes, para la operación de las actividades laborales, por otra parte, tienen existe debilidad en el proceso de sistematización de los conocimientos para mejorar la disponibilidad de recursos para el estudio y aprendizaje. Según Bustos y Colb (2010) y Cabero et al (2018), la biblioteca universitaria debe ser un centro de información, de forma tal que su misión se cumplirá si realiza gestión de información. La gestión de información incluye selección, adquisición, arriendo, organización, control, diseminación y uso de información en todas sus variedades de fuentes y soportes, relevante para la efectiva operación de las organizaciones.

\section{DISICUSION FINAL}

Las tecnologías de información y comunicación utilizadas como plataforma de servicio son los medios de almacenamiento, los cuales cumplen los requerimientos, además las redes le ofrecen ventajas con respecto a la rapidez para obtener información. En ellas se cuentan con una infraestructura de datos para respaldar la información en la biblioteca, en virtud de las labores ejecutadas por el personal en forma coordinada con el desarrollo tecnológico y las bases de datos que manejan las bibliotecas, de las instituciones en estudio. En este sentido se asocia la información propuesta por Viloria et al (2019), quienes manifiestan como una necesidad, que las bibliotecas universitarias contar con colecciones digitales, facilidades de acceso a otras colecciones, es decir las que tiene la institución y las que reposan en otras instituciones, adquisiciones cooperativas a través de consorcios, almacenamiento cooperativo, acceso electrónico a bases de datos y a otros recursos de información disponibles a través de las redes de telecomunicaciones. 
El hecho más relevante, es que las bibliotecas universitarias, se conciben como centros de información digital que en la actualidad se ha ido modernizando para dar respuesta a los usuarios, tales como estudiantes y docentes. Destacando que, con el uso de base de datos electrónicas, se puede generar y desarrollar información en todas sus variedades de fuentes, para la operación de las actividades laborales, por otra parte, cuentan con procesos de sistematización de los conocimientos para mejorar la disponibilidad de recursos para el estudio y aprendizaje. De acuerdo con Bustos y Colb, (2010), la base de datos electrónica debe incluir selección, adquisición, arriendo, organización, control, diseminación y uso de información en todas sus variedades de fuentes y soportes, relevante para la efectiva operación de las organizaciones. En este sentido, se destaca que la gestión de información contempla desarrollar equilibrios entre recursos de información actual, además permanente, entre información local y virtual, entre recursos de información propios, así como el acceso a recursos contra demanda en el momento que se requieren, en la diversificación de soportes de información analógicos y digitales, mono y multimediales.

Tambien se evidencia que se debe promover el desarrollo de las competencias investigativas en función de asumir el proceso de análisis de los recursos integrados a la tecnología digital, tanto del personal que promueve la atención en las bibliotecas, como estudiantes y docentes. Cai (2017), sostiene que el personal de las bibliotecas debe estar continuamente actualizados, para apoyar el usuario en el área de la informática, por otro lado, se deben utilizar diversos recursos tecnológicos en los procesos investigativos.

En otro orden de ideas, el nivel de información está relacionado con las actividades, se concluye que las mismas están orientadas a la trasmisión efectiva de la información en las bibliotecas, aunque en oportunidades no se vinculan ciertos elementos, datos que, relacionados con el manejo de las TIC, disminuye el proceso de desarrollo tecnológico, Tarute y Gatautis (2014), expresan que se requiere la existencia de una infraestructura de datos, promoviendo que pudiese haber un margen de no manejo del proceso, además de pocas habilidades para revisar los procedimientos técnicos utilizados para proveer el servicio al usuario, esto indica que algunos de los empleados de atención al usuario, no tienen la competencia para revisar las computadoras en caso de alguna necesidad, por ejemplo, instalar algún dispositivo.

\section{CONCLUSIONES}

De acuerdo al trabajo presentado, a los resultados obtenidos y a su discusión y análisis, se pueden plantear las siguientes conclusiones principales:

1.- Las instituciones universitarias Universidad del atlántico y Universidad de Sucre de la Region Caribe Colombiana tienen la responsabilidad de adaptarse a las tendencias globales, poniendo a disposición de los integrantes de la comunidad académica los recursos necesarios para preservar y difundir el conocimiento con el uso adecuado de las estrategias tecnológicas digitales.

2. Los medios de almacenamiento, las redes y las bases de datos electrónicas, como el conjunto de estrategias tecnológicas, se han convertido en un conjunto de herramientas que abre nuevas posibilidades a las bibliotecas y a sus profesionales, generando la oportunidad de capturar información en cualquier parte del mundo favoreciendo las conexiones a través de redes, de esta manera responden al reto del mundo de estrategias digitales que fundamentan los recursos investigativos.

3.- El estudio ha permitido esclarecer que en las bibliotecas universitarias de la universidad del Atlantico y universidad de Sucre, cuentan con una serie de recursos electrónicos a disposición de los usuarios, cuentan con colecciones digitales, y están organizando repositorios, ademas de actualizacion de procesos, donde el experto en biblioteca guía a los usuarios hacia las fuentes más relevantes, abriendo camino al conocimiento, a la información incluida en las líneas de la información.

Se considera que en las universidades se debe organizar de forma efectiva los recursos digitales, los medios de almacenamientos, las redes y las bases de datos, de tal manera de promover el acceso fácil a los datos en línea, al mismo tiempo enseñar a los usuarios cómo utilizar eficazmente la información la electrónica en el desarrollo de sus investigaciones.

\section{REFERENCIAS}

Berrio, S., Pitre, R., y Hernandez, H., Impact of ICT on the Generation of New Services Companies, doi: https://doi.org/10.12988/ces.2018.86272, Contemporary Engineering Sciences, 11(52), 2591-2599 (2018)

Bosco, A., y Rodríguez, D., Virtual university teaching: contributions to innovation in higher education. The case of Online Geography at the Universitat Autònoma de Barcelona. doi: https://doi.org/10.1080/14703297.2010.543772, Innovations in Education and Teaching International, 48(1), 13-23, (2011) 
Cai, Y., From an analytical framework for understanding the innovation process in higher education to an emerging research field of innovations in higher education. doi: https://doi.org/10.1353/rhe.2017.0023, The Review of Higher Education, 40(4), 585-616 (2017)

Dirks, L., y Hey, T. The Coming Revolution in Scholarly Communications \& Cyberinfrastructure. CT Watch Q, 3(3), (2007)

Bustos, A., y Colb, C., Los entornos virtuales como espacios de enseñanza y aprendizaje. Una perspectiva psicoeducativa para su caracterización y análisis, http://www.scielo.org.mx/scielo.php?script=sci_arttext\&pid=S140566662010000100009, ISSN: 1405-6666, Revista Mexicana de Investigación Educativa, 15(44), 163-184 (2010)

Cabero, J., Vázquez, E., y López, E., Uso de la Realidad Aumentada como Recurso Didáctico en la Enseñanza Universitaria, doi: https://dx.doi.org/10.4067/S0718-50062018000100025, Formación universitaria, 11(1), 25-34 (2018)

Hernández, L., Balmaceda, N., y otros 5 autores, Optimization of a WiFi Wireless Network that Maximizes the Level of Satisfaction of Users and Allows the Use of New Technological Trends in Higher Education Institutions, doi: https://doi.org/10.1007/978-3-030-21935-2_12, In International Conference on Human-Computer Interaction , 144-160, Springer, Cham (2019)

Hernández L., Jiménez G., y otros 3 autores, Characterization of the Use of the Internet of Things in the Institutions of Higher Education of the City of Barranquilla and Its Metropolitan Area. In: Stephanidis C. (eds) HCl International $2018-$ Posters' Extended Abstracts. HCl 2018. Communications in Computer and Information Science, ISSN: 1865-0929, 852. Springer, Cham (2018)

Krull, G., y Duart, J.M., Research trends in mobile learning in higher education: A systematic review of articles (20112015), doi: https://doi.org/10.19173/irrodl.v18i7.2893, International Review of Research in Open and Distributed Learning, 18(7), (2017)

Mačiulytè-Šniukienè, A., y Gaile-Sarkane, E., Impact of information and telecommunication technologies development on labour productivity, doi: https://doi.org/10.1016/j.sbspro.2013.12.974, Procedia-Social and Behavioral Sciences, 110(26), 1271-1282 (2014)

Manly, T.S., Leonard, L.N., y Riemenschneider, C.K., Academic integrity in the information age: Virtues of respect and responsibility, doi: https://doi.org/10.1007/s10551-014-2060-8, Journal of Business Ethics, 127(3), 579-590 (2015)

Martínez, O., Steffens, E., Ojeda, D., y Hernández, H., Estrategias Pedagógicas Aplicadas a la Educación con Mediación Virtual para la Generación del Conocimiento Global, doi: https://dx.doi.org/10.4067/S0718-50062018000500011, Formación universitaria, 11(5), 11-18 (2018)

Martínez, O., Steffens, E., Ojeda, D., García, J., y otros 2 autores, Niveles de pensamiento crítico en estudiantes de Universidades Región Caribe (Colombia), http://www.revistaespacios.com/a17v38n30/a17v38n30p05.pdf, ISSN: 07981015, Revista espacios, 38(30) (2017)

Mercado, D., Sepúlveda, J., Pedraza, L., y Hernández, H., Modelo de implementación de TIC en el sector transporte de la ciudad de Barranquilla utilizando dinámica de sistemas, doi: https://doi.org/10.15665/rde.v12i1.69, Dimensión empresarial, 12(1), 36-45 (2014)

Ng'ambi, D., y Bozalek, V., Emerging technologies and changing learning/teaching practices, doi: https://doi.org/10.1111/bjet.12061, British Journal of Educational Technology, 44(4), 531-535 (2013)

Novo-Corti, I., Varela-Candamio, L., y Ramil-DíAz, M., E-learning and face to face mixed methodology: Evaluating effectiveness of e-learning and perceived satisfaction for a microeconomic course using the Moodle platform, doi: https://doi.org/10.1016/j.chb.2012.06.006, Computers in Human Behavior, 29(2), 410-415 (2013)

Sánchez, M., García, J., Steffens, E., y Hernández, H., Estrategias Pedagógicas en Procesos de Enseñanza y Aprendizaje en la Educación Superior incluyendo Tecnologías de la Información y las Comunicaciones, doi: https://dx.doi.org/10.4067/S0718-07642019000300277, Información tecnológica, 30(3), 277-286 (2019)

Sangrá, A., La calidad en las experiencias virtuales de educación superior (2010)

Tarute, A., y Gatautis, R., ICT impact on SMEs performance, doi: https://doi.org/10.1016/j.sbspro.2013.12.968, Procedia Social and Behavioral Science, 110, 1218-1225 (2014)

Villarreal, S., García, J., Hernández, H., y Steffens, E., Competencias Docentes y Transformaciones en la Educación en la Era Digital, doi: https://dx.doi.org/10.4067/S0718-50062019000600003, Formación universitaria, 12(6), 3-14 (2019)

Viloria, A., Rodríguez, J., Payares, K., Vargas, C. y otros 3 autores, Determinating Student Interactions in a Virtual Learning Environment Using Data Mining, doi: https://doi.org/10.1016/j.procs.2019.08.082, Procedia Computer Science, 155, 587$592(2019)$ 